$01,10,11$

\title{
Вырожденная структура двойников превращения и оценка плотности дислокаций мартенситных кристаллов
}

\author{
(C) М.П. Кащенко ${ }^{1,2}$, Н.М. Кащенко ${ }^{1}$, В.Г. Чащина ${ }^{1,2}$ \\ ${ }^{1}$ Уральский федеральный университет, \\ Екатеринбург, Россия \\ ${ }^{2}$ Уральский государственный лесотехнический университет, \\ Екатеринбур, Россия \\ E-mail: mpk46@mail.ru
}

Поступила в Редакцию 16 июля 2019 г.

В окончательной редакции 16 июля 2019 г.

Принята к публикации 25 июля 2019 г.

В динамической теории мартенситных превращений волновой механизм управления ростом кристаллов мартенсита обусловлен наложением волновых пучков квазипродольных (либо продольных) волн, несущих деформацию типа „растяжение-сжатие“ в ортогональных направлениях. Появление волновых пучков считается следствием возникновения начальных возбужденных (колебательных) состояний.

Наличие двойников превращения интерпретируется как результат согласованного распространения относительно длинноволновых ( $l$-волны) и коротковолновых $(s$-волны) смещений. Условие согласования анализируется для мартенситного превращения $\gamma-\alpha$ в сплавах на основе железа. Впервые показано, что переход к вырожденной двойниковой структуре с учетом дискретности среды позволяет выполнить оценку плотности дислокаций в кристаллах с габитусами $\{557\}$, согласующуюся с экспериментально наблюдаемой.

Ключевые слова: мартенситные превращения, двойники превращения, управляющий волновой процесс, габитусные плоскости, вырожденная двойниковая структура, плотность дислокаций.

DOI: $10.21883 /$ FTT.2019.12.48532.04ks

\section{1. Введение}

Мартенситное превращение (МП) реализуется во многих кристаллических материалах, имеет кооперативный характер и протекает, как правило, с признаками фазового перехода первого рода. Попытки интерпретации гетерогенного зарождения на основе традиционных представлений о существовании квазиравновесных зародышей новой фазы, с одной стороны, не подкрепляются надежными наблюдениями подобных зародышей (проблема ненаблюдаемости зародышей), а с другой стороны, не позволяют адекватно интерпретировать богатую совокупность наблюдаемых особенностей МП.

Особенно выпукло эти проблемы проявляются при попытке описания ГЦК-ОЦК (ОЦТ) превращения в сплавах железа. Именно изучение этого превращения (далее для краткости МП $\gamma-\alpha$ ) способствовало накоплению наибольшей информации, положенной в основу определения мартенситного превращения как специфического (бездиффузионного) кооперативного превращения [1]. Наиболее яркой особенностью спонтанного (при охлаждении) МП $\gamma-\alpha$ является сверхзвуковая (по отношению к продольным волнам) скорость роста кристаллов мартенсита. Это обстоятельство сразу позволяет исключить из рассмотрения дислокационные модели роста кристаллов, безальтернативно указывая на волновую природу управления ростом кристаллов. Таким образом, следовало прояснить специфику гетерогенного старта роста кристаллов, приводящего к возникновению волно- вого процесса, управляющего формированием кристалла и позволяющего интерпретировать наблюдаемый набор морфологических признаков. Эта программа была выполнена в ходе развития динамической теории МП [2-4].

Центральную роль при этом играет концепция начального возбужденного (колебательного) состояния (НВС). НВС возникает в упругих полях отдельных дислокаций, имеет форму вытянутого параллелепипеда с ориентациями ребер, близкими к ориентациям собственных векторов $\xi_{1}, \xi_{2}$ и $\xi_{3}$ тензора деформации упругого поля дислокационного центра зарождения (ДЦЗ). Причем в области, благоприятной для возникновения НВС, два собственных значения тензора деформации имеют разные знаки, а третья деформация мала $\left(\varepsilon_{1}>1, \varepsilon_{2}<0\right.$, $\left.\varepsilon_{3} \ll\left|\varepsilon_{1,2}\right|\right)$. Абсолютные значения $\left|\varepsilon_{1,2}\right|$ считаются сравнимыми с пороговыми величинами межфазных барьеров $\varepsilon_{t h} \sim 10^{-4}-10^{-3}$. Тогда при охлаждении до температуры $M_{s}$ (ниже температуры равновесия фаз $T_{0}$ ) в области со сниженным значением пороговой деформации скачком возникает НВС при согласованном быстром скачке атомов к новым положениям равновесия с возбуждением колебаний в ортогональных направлениях, близких к $\xi_{1}$, $\xi_{2}$. Подобные колебания порождают волновые пучки с ортогональными волновыми векторами и скоростями $\mathbf{v}_{1}$, $\mathbf{v}_{2}$. Причем существенна область наложения волновых пучков, в которой реализуется пороговая деформация типа „растяжение-сжатие“ (см. рис. 1).

Как следует из рис. 1, плоским границам кристалла (границам раздела фаз-габитусам) в такой модели со- 


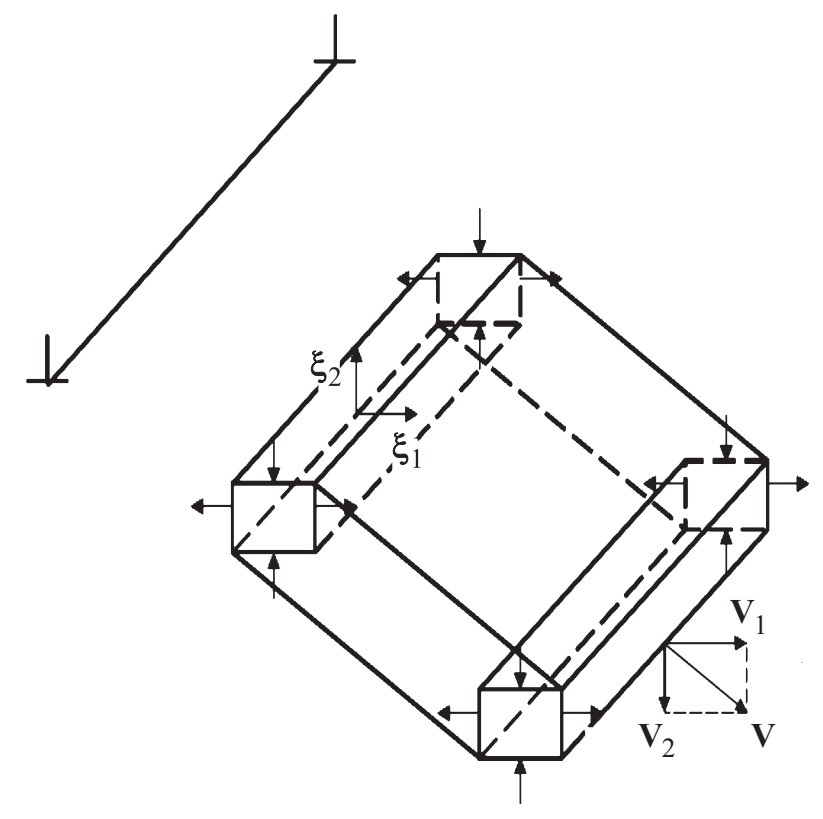

Рис. 1. Двухволновая схема формирования пластинчатого мартенситного кристалла [3].

ответствует движение со скоростью $\mathbf{v}=\mathbf{v}_{1}+\mathbf{v}_{2}$ линии пересечения фронтов волновых пучков. Таким образом, возникновение НВС порождает простейший вариант управляющего волнового процесса (УВП). Далее эту пару квазипродольных волн с относительно большими длинами волн будем именовать $l$-волнами. Легко показать, что ориентация нормали $\mathbf{N}$ к габитусной плоскости задается соотношением

$$
\begin{gathered}
\mathbf{N}_{1,2 l} \| \mathbf{n}_{2 l} \pm \varkappa_{l l} \mathbf{n}_{1 l}, \quad \varkappa_{l l}=\frac{v_{2 l}}{v_{1 l}}, \quad\left|\mathbf{n}_{1,2 l}\right|=1 \\
\mathbf{n}_{1 l}=\frac{\mathbf{v}_{1 l}}{v_{1 l}}, \quad \mathbf{n}_{2 l}=\frac{\mathbf{v}_{2 l}}{v_{2 l}}
\end{gathered}
$$

Однако мартенситные кристаллы могут обладать и тонкой структурой двойников превращения. Двойники превращения возникают непосредственно в ходе роста мартенситных кристаллов в виде чередующихся пластинчатых компонент. Они наблюдаются как в случае ярко выраженных признаков фазовых переходов I рода (например, в сплавах железа [1]), так и при МП, близких к фазовым переходам II рода (например, в ряде сплавов цветных металлов [5]). Типичное различие компонент двойниковой структуры (ДС) заключается в различии ориентаций главных осей деформации, как правило, ортогональных друг другу в исходной фазе.

При сравнительно низких (дозвуковых) скоростях роста мартенситных кристаллов в качестве механизма роста допустимо использование дислокационных представлений при трактовках ДС (см., например, [6]). При высоких же скоростях роста кристаллов, обладающих ДС, естественно опираться на динамические (волновые) трактовки, использующие концепцию УВП. В предыдущих работах было показано, что концепция УВП, после включения в структуру УВП более коротких $s$-волн, позволяет описать не только идеальную (строго регулярную) ДС $[3,7,8]$, но и, базируясь на модели формирования регулярной ДС, ставить и решать задачу интерпретации реальных неоднородных вариантов ДС [9-13]. Одним из принципиальных итогов такого анализа является вывод о фрагментации ДС, причем каждый фрагмент порождается единственной спонтанно возникшей возбужденной ячейкой, инициирующей $s$-волны. Динамическая теория позволяет рассмотреть и предельный случай вырожденной двойниковой структуры (ВДС), в которой доминирует основная компонента [14].

Хорошо известно [1], что среди наблюдаемых при МП $\gamma-\alpha$ в сплавах железа морфотипов, два варианта кристаллов с габитусами близкими к $\{225\}$ и $\{259\}-\{31015\}$ (кристаллографические обозначения приводятся в базисе исходной $\gamma$-фазы) имеют частично или полностью двойникованную центральную часть. Напротив, кристаллы с габитусами, близкими к семейству $\{557\}$, подобной двойниковой структурой не обладают, но демонстрируют высокую плотность дислокаций в мартенсите. Поэтому закономерно возникал вопрос о причинах такого различия. Цель настоящей работы показать, что переход к ВДС может сопровождаться формированием дислокаций, и провести оценку плотности дислокаций на конкретном примере кристаллов с габитусами, близкими к $\{557\}$.

\section{2. Основные представления о возникновении ДС и ВДС}

Напомним, что при МП $\gamma-\alpha$ двойники превращения контактируют по плоскостям $\{110\}$. Такие плоскости задают пары $s$-волн, распространяющихся вдоль ортогональных осей $\langle 100\rangle$ и $\langle 010\rangle$ исходной фазы (направления $\Delta$ в первой зоне Бриллюэна) со скоростями $v_{s \Delta}$. Действительно, ориентации нормалей

$$
\mathbf{N}_{1,2, s} \|[110],[1 \overline{1} 0]
$$

к плоскостям двойникования тривиально находятся из (1) при заменах

$$
\varkappa_{l l} \rightarrow \varkappa_{s s}=1, \mathbf{n}_{1 l} \rightarrow \mathbf{n}_{1 s}=[010], \mathbf{n}_{2 l} \rightarrow \mathbf{n}_{2 s}=[100] .
$$

Механизм снятия вырождения по ориентациям плоскостей двойникования подробно рассмотрен в [3]. Если возникновение НВС, инициирующего $l$-волны, сопровождается более или менее синхрониированным возникновением внутри области локализации НВС и возбужденной области в форме тонкого колеблющегося параллелепипеда, инициирующего возбуждение пар $s$-волн, то результирующий УВП позволяет корректно описать формирование тонкопластинчатых двойникованных кристаллов (или двойникованных центральных областей 


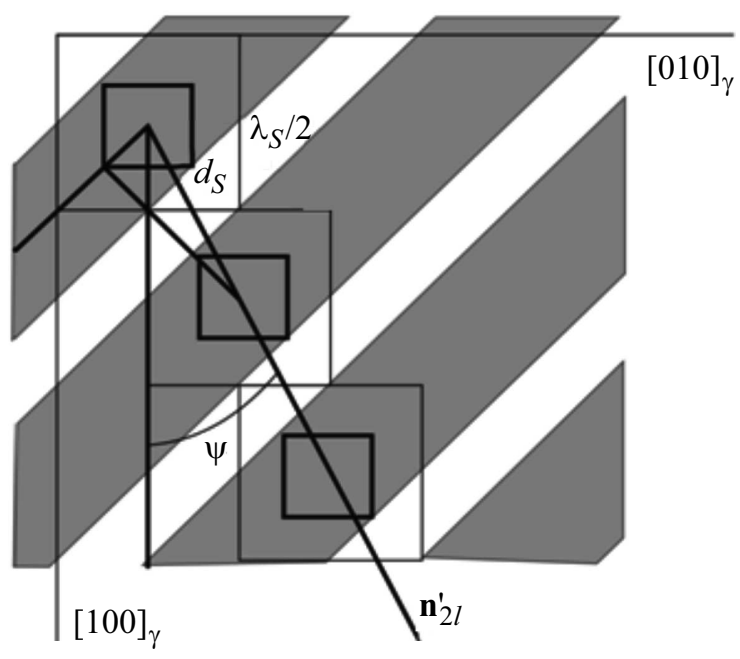

Рис. 2. Динамическая модель формирования регулярной слоистой структуры с соотношением долей компонент 2/1 [12].

линзовидных кристаллов). Причем основные компоненты ДС физически выделены, так как именно они инициируются действием волн, двойниковые же прослойки возникают в силу когерентной связи контактирующих областей решетки.

Процесс индуцированного воспроизводства возбужденной $s$-ячейки реализуется после прохождения за одно и то же время двух катетов треугольника в направлениях [1ํㅣ и [110] суперпозицией $s$-волн, а гипотенузы треугольника - $l$-волной, несущей деформацию сжатия, со скоростью, равной проекции $v_{2 l}^{\prime}$ скорости $v_{2 l}$ на плоскость (001) (см. рис. 2). При скорости прохождения катетов, равной $\sqrt{2} v_{s \Delta}$, получаем условие

$$
v_{s \Delta}=v_{2 l}^{\prime} \cos \psi,
$$

где $\psi$ острый угол между $n_{2 l}^{\prime}$ и $v_{s \Delta} \|\langle 001\rangle_{\gamma}$. При гармоническом описании пороговой деформации считается, что потеря устойчивости решетки исходной фазы происходит в области с поперечным размером $d_{s}<\lambda_{s} / 2$, где $\lambda_{s}$ - длина $s$-волны. Тогда для отношения $\beta_{t w}$ объемов компонент ДС находим

$$
\beta_{t w}=4 \widetilde{d}_{s} /\left(1+\operatorname{tg} \psi-4 \widetilde{d}_{s}\right), \quad \widetilde{d}_{s}=d_{s} / \lambda_{s}<1 / 2 .
$$

Согласно (5), случай ВДС соответствует выполнению условия

$$
1+\operatorname{tg} \psi-4 \widetilde{d}_{s}=0 .
$$

Такой вариант, ведущий к сингулярности $\beta_{t w} \rightarrow \infty$, связан с континуальным описанием, не учитывающим дискретность кристаллической среды. Когерентность сопряжения компонент, как очевидно из рис. 3, указывает на возможность снятия сингулярности путем рождения дислокации на месте двойниковой прослойки.

Действительно, минимальная толщина слоя между основными компонентами ДС равна расстоянию между ближайшими атомными плоскостями (110), то есть $a / \sqrt{2}$, где $a$ - параметр ГЦК-решетки. Если относительное смещение соседних плоскостей достигнет такой же величины, соответствующей вектору Бюргерса полной дислокации, естественно ожидать рождения дислокационной петли, основные сегменты которой имеют винтовую ориентацию. Ясно, что величина деформации сдвига в двойниковой компоненте при этом составляет $\operatorname{tg} \varphi_{t w}=1$. Поскольку двойниковая компонента находится в обрамлении пары основных компонент ДС, каждая из основных компонент вносит в относительное смещение соседних плоскостей вклад $a 2 / \sqrt{2}$. Ранее было показано [3], что при двойниковании первый быстрый этап деформации связан с действием $s$-волн, причем при равенстве скоростей следует ожидать и равенства величин деформаций сжатия и растяжения: $\varepsilon_{1 s}=\left|\varepsilon_{2 s}\right|=\varepsilon_{s}$. Равенство относится не только к пороговым $\varepsilon_{\text {sth }}$, но и к финишным значениям деформации первого этапа $\varepsilon_{\mathrm{sf}}$.

Это означает, что и величина деформации результирующего сдвига в основной компоненте ДС, имеющей толщину $d_{s} \sqrt{2}$, составляет $\operatorname{tg} \varphi_{b a s}=\varepsilon_{s f}$. Отсюда следует, что рождение дислокаций уже на первом этапе деформации возможно при выполнении условия

$$
d_{s} \sqrt{2} \varepsilon_{s f}=a / 2 \sqrt{2}, \quad d_{s} \varepsilon_{s f}=a / 4
$$

Напомним [3]: в случае, когда двойниковая компонента реально образуется, естественно полагать, что финишная бейновская деформация сжатия $\left(\left|\varepsilon_{2 \mathrm{~B}}\right| \leq 0.2\right)$ в двойниковой области достигается уже на первом этапе деформации, тогда как в основной компоненте может быть достигнута лишь меньшая деформация растяжения. В случае с ВДС вместо двойниковой мартенситной прослойки в намеченной УВП слоистой структуре рождаются дислокации, что требует несколько меньших, чем $\left|\varepsilon_{2 \mathrm{~B}}\right|$ (но того же порядка), деформаций $\varepsilon_{s f}$. Уточнение величины $\varepsilon_{s f}$ возможно, если учесть, что, согласно (5),

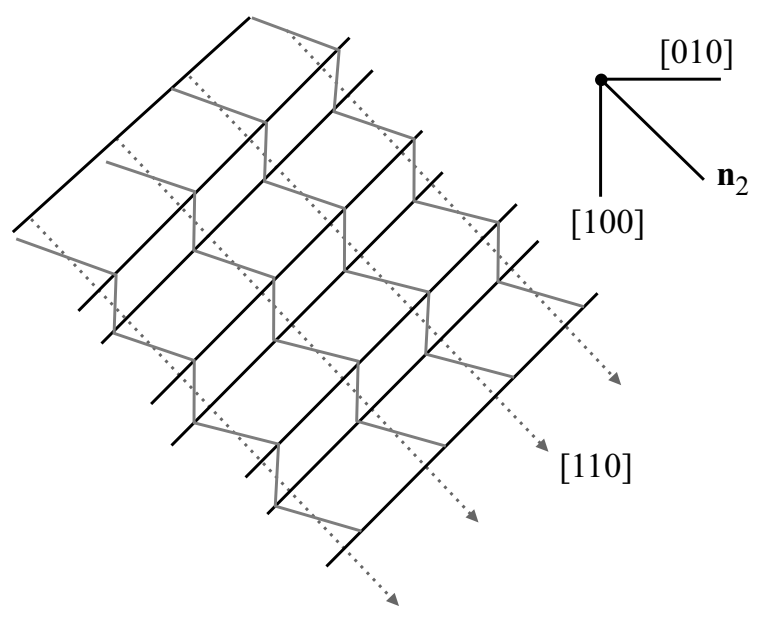

Рис. 3. Система чередующихся сдвигов в основной и дополнительной компонентах регулярной слоистой структуры при отношении объниов компонент 2/1 [3]. 
максимальное значение $d_{s \max }=\lambda_{s} / 2$. Значит, определение величины $\lambda_{s}$ из дополнительного условия позволило бы с помощью (7) найти и значение $\varepsilon_{s f}$. Таким дополнительным условием является соотношение (4) для скоростей $s$ - и $l$-волн.

\section{3. Выполнение условия согласования скоростей $s$ - и $l$-волн и оценка плотности дислокаций}

Заметим, что для двойникования в кристаллах с габитусами (3 1015$)-(259)$ условие (4), оптимальное для формирования регулярной ДС, точно не выполняется при наблюдаемых упругих модулях. Более того, оно не может быть выполнено и за счет учета дисперсии, снижающей $v_{s}$ при росте квазиимпульса. Действительно, в плоскости симметрии кубической решетки (001) в произвольном направлении $[\cos \psi, \sin \psi, 0]$, составляющем угол $\psi$ с осью $[100]$, в длинноволновом пределе для отношения скоростей продольных волн, согласно уравнению Кристоффеля [15], имеет место равенство.

$$
\frac{v(\psi)}{v_{\Delta}}=\sqrt{1+\left(\frac{C_{L}}{C_{11}}-1\right)(\sin 2 \psi)^{2}},
$$

где $C_{L}$ и $C_{11}$ - упругие модули, задающие скорости продольных волн в направлениях осей симметрии второго $\left(v_{\Sigma}\right)$ и четвертого $\left(v_{\Delta}\right)$ порядка. Легко убедиться, что при подстановке в (8) значений измеренных упругих модулей [16] в области сравнительно малых углов

$$
\frac{v(\psi)}{v_{\Delta}}>\frac{v(\psi)}{v_{s}}=\frac{1}{\cos (\psi)} .
$$

Для кристаллов с габитусами, близкими к $\{31015\}_{\gamma}-\{259\}_{\gamma}$, угол $\psi$ меняется от $\sim 16.7^{\circ}$ до $\sim 21.8^{\circ}$, и неравенство (9) выполняется, усиливаясь при переходе к $v_{s}<v_{\Delta}$. Поэтому регулярной ДС и не наблюдается. Напротив, при стремлении $\psi$ к $\pi / 4$

$$
\frac{v(\psi)}{v_{\Delta}}<\frac{v(\psi)}{v_{s}}=\frac{1}{\cos (\psi)} \text {. }
$$

Значит, снижение скорости $v_{\Delta}$ при переходе к $v_{s}<v_{\Delta}$ приведет к равенству (4).

Уместно отметить, что в динамической теории наличие габитусов $\{557\}$ легко объясняется [2] (включая и небольшое различие в значениях пары индексов) как следствие распространения пар $l$-волн вблизи пар ортогональных направлений $\langle 110\rangle$ и $\langle 001\rangle$, но возможность включения s-волн в состав УВП для этого случая ранее не учитывалась. Поскольку $\langle 110\rangle$ составляет с $\langle 101\rangle$ угол $\pi / 4$, описание кристаллов с габитусами $\{557\}$ связано, в случае ВДС, фактически с требованием

$$
v_{s \Delta}=v_{2 l}^{\prime} \cos \pi / 4 \approx v_{\Sigma} / \sqrt{2} .
$$

Найдем квазиимпульс $s$-волны, при котором выполняется требование (11). Для этого используем найденную ранее аналитическую интерполяцию закона дисперсии фононов $\varepsilon_{k}$ в $\Delta$-направлении [10], хорошо согласующуюся как с измерениями скоростей звука [16], так и с данными нейтронных исследований [17]. Закон дисперсии $\varepsilon_{k}$ вдоль $\langle 001\rangle_{\gamma}$ для $0 \leq k \leq k_{\max }=2 \pi / a(a-$ параметр решетки) аппроксимируется в безразмерных переменных $y$ и $x$ функцией

$$
1-y=(1-x)^{p}, \quad y=\varepsilon_{k} /\left(\varepsilon_{k}\right)_{\max }, \quad x=k / k_{\max } .
$$

Например, для сплава $\mathrm{Fe} 30 \mathrm{Ni}$ с ГЦК решеткой соответствие с экспериментальными данными достигается при $p \approx 1.733$. Для групповых скоростей $v_{g}(x)=d y / d x$ $s$-волн имеем:

$$
\begin{gathered}
v_{s g}(x)=d y / d x=p(1-x)^{p-1}, \quad v_{g}(0)=v_{\Delta}=p, \\
v_{s g}(x) / v_{g}(0)=v_{s g}(x) / p=(1-x)^{p-1} .
\end{gathered}
$$

Фактически нас интересует отношение

$$
\begin{aligned}
v_{s g}(x) / v_{\Sigma} & =\left(v_{s g}(x) / v_{\Delta}\right)\left(v_{\Delta} / v_{\Sigma}\right) \\
& =(1-x)^{p-1} \sqrt{\frac{C_{11}}{C_{L}}} .
\end{aligned}
$$

Подставляя в (14) $C_{11}=0.1745, C_{L}=0.2508 \mathrm{TPa}$, согласно данным [16] для Fe-31.5 при температуре $673 \mathrm{~K}$, и требуя

$$
v_{s g}(x) / v_{\Sigma}=(1-x)^{p-1} \sqrt{\frac{C_{11}}{C_{L}}}=\frac{1}{\sqrt{2}},
$$

при $p=1.733$ находим $x \approx 0.2$, то есть $q_{s} \approx 0.2 q_{\max }$ $=0.4 \pi / a$. Значит, $d_{s \max }=\lambda_{s} / 2=2.5 a$.

Тогда из (7) получаем $\varepsilon_{s f}=0.1$. Таким образом, на первом этапе $s$-деформация достигает величины $\varepsilon_{s}=0.1$. Допускаем, что в прослойке между соседними основными компонентами ВДС рождается только одна дислокационная петля, давая в сечении пару линий. Из геометрии зарождения реечного кристалла следует, что эти линии направлены вдоль вытянутой оси исходного HВC, то есть при габитусе (557) вдоль [110]. Этот вариант отражает рис. 4.

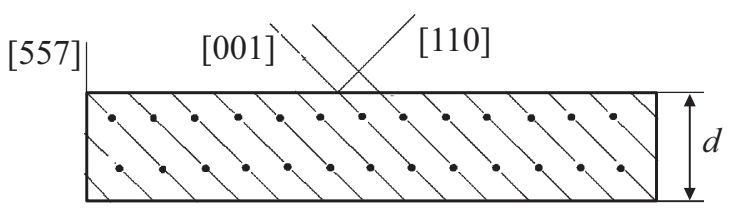

$[77 \overline{10}]$

Рис. 4. Схема сечения кристалла с ВДС, имеющего габитус (557), плоскостью (11̄0): пары точек на линиях [001] следы основных сегментов дислокационных петель в плоскостях (110) с винтовой ориентацией вектора Бюргерса; расстояние между плоскостями соседних петель равно $d_{s} \sqrt{2}$. 
Тогда для плотности дислокаций $\rho$ имеем:

$$
\rho \approx 2 /\left(2 d_{s} / d\right)=1 /\left(d_{s} / d\right) .
$$

При толщине кристалла $d=1 \mu \mathrm{m}=10^{-4} \mathrm{~cm}$, $d_{s}=2.3 a$ и $a \approx 3.5 \cdot 10^{-8} \mathrm{~cm}$ получаем оценку $\rho \approx 1.14 \cdot 10^{11} \mathrm{~cm}^{-2}$, которая коррелирует с экспериментальными данными $[1,18,19]$.

\section{4. Обсуждение результатов}

Прежде всего отметим, что кристаллы с габитусами, близкими $\{557\}$, имеют вид „реек“ то есть явно неравноосных кристаллов. Ансамбли таких кристаллов образуют специфические колонии, названные „пакетами“ (из 24 возможных вариантов кристаллы в каждом пакете могут содержать не более шести [19]). Таким образом, представляет интерес объяснение особенностей формирования как отдельного кристалла, так и всего ансамбля пакетного мартенсита. С позиций минимизации упругой энергии, качественная трактовка сводится к тому, что группировки кристаллов в пакеты отражают варианты аккомодации внутренних напряжений.

Кроме того, кристаллы с габитусами $\{557\}$ образуются при относительно высоких температурах, и релаксация возникающих напряжений осуществляется сдвигами в окружающий остаточный аустенит. Однако высокую плотность дислокаций внутри мартенсита это не объясняет.

В динамической теории особенности формирования пакета кристаллов естественно интерпретируются как самоорганизующийся процесс волнового роста отдельных кристаллов в упругих полях ДЦЗ, последовательное появление которых инициируется растущими кристаллами $[20,21]$. Выше уже отмечалось, что наличие габитусов $\{557\}$ является следствием распространения пар $l$-волн вблизи ортогональных направлений $\langle 110\rangle$ и $\langle 001\rangle$. Поскольку деформация Бейна трехмерна, то, без учета в составе УВП $s$-волн, допускалось, что деформация в третьем ортогональном направлении осуществляется за счет электронных корреляций, восстанавливающих симметрию электронной плотности. В [21] появление наблюдаемой плотности дислокаций связывается с процессами распространения и частичной аннигиляции кристонов - объемных носителей деформации сдвига супердислокационного типа.

Интересен и вариант [22], интерпретирующий возникновение кристаллов как процесс деформации плоскостей $\{110\}$. Однако наиболее предпочтительной, на наш взгляд, является предлагаемая здесь трактовка появления дислокаций, как следствия развития ВДС в условиях согласованности $s$ - и $l$-волновых деформаций. Действительно, такой механизм МП сразу обеспечивает трехмерную деформацию, снимая неопределенность при выборе ориентации главной оси сжатия, приводя к общему для габитусов $\{h k l\}$ правилу. А именно: при $|h|<|k|<|l|$ ориентации главной оси сопоставляется направление $\langle 010\rangle$ с позицией единицы, соответствующей позиции среднего индекса $k$ в записи габитуса. Позиции наибольшего индекса 1 сопоставляется направление растяжения $\langle 001\rangle$. Напомним, что запись габитуса $\{557\}$ с равенством пары индексов оправданна, если обе $l$-волны лежат в плоскости $\{1 \overline{1} 0\}$. Этот вариант возможен при возникновении НВС в упругих полях краевых дислокаций с линиями $\langle 1 \overline{1} 0\rangle$. Однако в случае 60-градусных дислокаций, появление НВС приведет к выходу волнового вектора $l$-волны, несущей деформацию сжатия, из плоскости $\{1 \overline{1} 0\}$. Это приводит к отклонению $\mathbf{n}_{2 l}$ от направления $\langle 110\rangle$ в плоскости $\{001\}$ и, соответственно, к нарушению равенства пары индексов, отличию $\psi$ от $\pi / 4$ и выбору в качестве главной оси сжатия той из осей $\langle 100\rangle$ или $\langle 010\rangle$, которая составляет меньший угол с $\mathbf{n}_{2 l}$. Ясно, что при $\psi<\pi / 4$ это ось $\langle 100\rangle$. Кроме того, при $\psi<\pi / 4$ условию (6) для перехода к ВДС соответствует неравентво $d_{s}<\pi / 4$, а значит, и ненулевая пороговая деформация.

Отметим также, что ранее отсутствие двойников превращения в реечных кристаллах рассматривалось как следствие высокого затухания $s$-волн. Однако прозрачная интерпретация механизма рождения дислокаций, опирающаяся на эффективность коротковолновых смещений, указывает на достаточное для реализации МП время их жизни. Это, скорее всего, связано с поддержкой $s$-волн неравновесной электронной подсистемой $[2,4,23]$. Ясно, что отклонения от выполнения условия (4) при спонтанном возникновении активных ячеек $s$ - $l$, порождающих $s$-волны, неизбежны. Уменьшение квазиипульсов $s$-волн будет сопровождаться ростом $\lambda_{s}$ и $d_{s}$, а значит, согласно (17), снижением плотности дислокаций. Напротив, уменьшение толщин $d$ ведет к повышению плотности $\rho$. Поэтому внутри пакета кристаллов следует ожидать значений $\rho$ из диапазона $10^{10}-10^{12} \mathrm{~cm}^{-2}$.

\section{5. Заключение}

Динамическая теория формирования регулярных слоистых структур при МП, включая двойники превращения, позволяет выполнить предельный переход к вырожденной структуре с учетом дискретности кристаллической среды.

Показано, что в случае формирования кристаллов с габитусами $\{557\}$ выполняется условие согласования для скоростей коротковолновых и длинноволновых смещений, позволяющее ввести регулярную структуру, в которой вместо двойниковых прослоек образуются дислокационные петли. Выполненная в такой модели оценка плотности дислокаций согласуется с экспериментально наблюдаемыми значениями.

\section{Благодарности}

Авторы признательны участникам XXI Зимней школы по механике сплошных сред (г. Пермь, 18-22 февраля 
2019г.) и международной конференции MGCTF-2019 (Санкт-Петербург, 1-5 июля 2019г.) за обсуждение результатов.

\section{Конфликт интересов}

Авторы заявляют, что у них нет конфликта интересов.

\section{Список литературы}

[1] Г.В. Курдюмов, Л.М. Утевский, Р.И. Энтин. Превращения в железе и стали. Наука, М. (1977). 240 с.

[2] М.П. Кащенко. Волновая модель роста мартенсита при $\gamma-\alpha$ превращении в сплавах на основе железа. Изд. 2-е. НИЦ „Регулярная и хаотическая динамика“. Ижевский институт компьютерных исследований, М.-Ижевск (2010). $280 \mathrm{c}$.

[3] М.П. Кащенко, В.Г. Чащина. Динамическая модель формирования двойникованных мартенситных кристаллов при $\gamma-\alpha$ превращении в сплавах на основе железа. Урал. гос. лесотехн. ун-т, Екатеринбург (2009). 98 с.

[4] М.П. Кащенко, В.Г. Чащина. УФН 181, 345 (2011).

[5] Х. Варлимонт, Л. Дилей. Мартенситные превращения в сплавах на основе меди, серебра и золота. Наука, М. (1980). $206 \mathrm{c}$

[6] М.А. Штремель. Прочность сплавов. Ч. ІІ. Деформация. МИСИС, М. (1997). 527 с.

[7] М.П. Кащенко, В.Г. Чащина, С.В. Вихарев. ФММ 110, 212 (2010).

[8] М.П. Кащенко, В.Г. Чащина, С.В. Вихарев. ФММ 110, 323 (2010).

[9] М.П. Кащенко, В.Г. Чащина. ФММ 114, 894 (2013).

[10] M.P. Kashchenko, I.F. Latypov, V.G. Chashchina. Lett. Mater. 7, 146 (2017).

[11] М.П. Кащенко, В.Г. Чащина. ФММ 118, 327 (2017).

[12] M.P. Kashchenko, N.M. Kashchenko, V.G. Chashchina. Mater. Today 4, 4605 (2017).

[13] М.П. Кащенко, Н.М. Кащенко, В.Г. Чащина. ФММ 119, 3 (2018).

[14] M.P. Kashchenko, N.M. Kashchenko, V.G. Chashchina. Lett. Mater. 8, 429 (2018).

[15] Ф.И. Федоров. Теория упругих волн в кристаллах. Наука, M. (1965). $388 \mathrm{c}$

[16] G. Haush, H. Warlimont. Acta Met. 21, 400 (1973).

[17] E.D. Hallman, B.N. Brockhouse. Can. J. Phys. 47, 1117 (1969).

[18] Т.В. Этерашвили, Л.М. Утевский, М.Н Спасский. ФММ 49, 807 (1979).

[19] Д.П. Родионов, В.М. Счастливцев. Стальные монокристаллы. УрО РАН, Екатеринбург. (1996). 275 с.

[20] В.П. Верещагин, М.П. Кащенко, С.В. Коновалов, Т.Н. Яблонская. ФММ 77, 173 (1994).

[21] М.П. Кащенко, В.В. Летучев, С.В. Коновалов, Т.Н. Яблонская. ФММ 83, 43 (1997).

[22] В.Г. Чащина. Изв. вузов. Физика 52, 95 (2009).

[23] М.П. Кащенко, Н.А. Скорикова, В.Г. Чащина. ФММ 106, 229 (2008).

Редактор Е.Ю. Флегонтова 